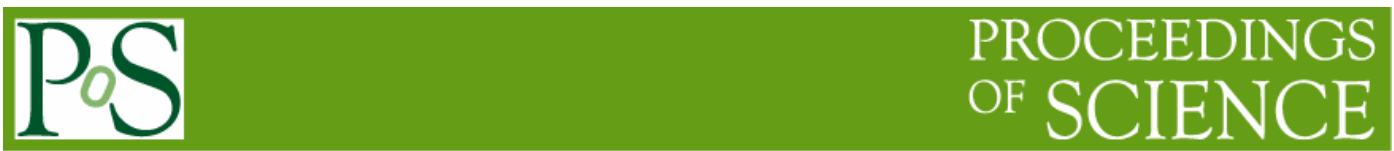

\title{
Monte Carlo Simulation of the Response Energy Distribution of Proton Recoil Proportional Counters
}

\author{
Richard Babut ${ }^{*}$ \\ Institute for Radiological Protection and Nuclear Safety \\ Cadarache BP3, 13115 Saint-Paul-lez-Durance, France \\ E-mail: richard.babut-arphytech@irsn.fr

\section{Vincent Gressier} \\ Institute for Radiological Protection and Nuclear Safety \\ Cadarache BP3, 13115 Saint-Paul-lez-Durance, France \\ E-mail: vincent.gressierairsn.fr
}

The Institute for Radiological Protection and Nuclear Safety uses proton recoil proportional counters as reference from some tenth of $\mathrm{keV}$ up to $2 \mathrm{MeV}$. To determine the neutron fluence energy distribution within a few percents uncertainties, the response matrices detector have to be accurately calculated.

The simulation codes used up to now to simulate the response energy distributions of the counters, present some limitations due to simplified geometry of the counters, electric field effect not always taken into account and (n,p) reactions of high energy neutrons in the counter walls not considered. The use of the Monte Carlo MCNPX code should provide a solution to reduce these limitations.

The electric field and gas amplification are calculated in a dedicated code before the generated effect, on the detector responses are calculated by MCNPX. The calculated responses of SP2 spherical proton recoil detectors are compared with experimental data obtained at reference mono-energetic fields and with the SPHERE code calculation. The importance of the proton ranges in the agreement with the experimental data will be discuted.

International Workshop on Fast Neutron Detectors

University of Cape Town, South Africa

April 3-6, 2006

\footnotetext{
${ }^{*}$ Speaker
} 


\section{Introduction}

To extend its set of neutron reference fields, the Institute for Radiological Protection and Nuclear Safety has a new accelerator called AMANDE [1].

The aim of this facility is to produce reference mono-energetic neutron fields, known both in energy and fluence, within the energy range $2 \mathrm{keV}-19 \mathrm{MeV}$. In a first step, two types of detectors will be used to determine the reference fluence energy distribution: a liquid scintillator BC501A [2] for neutron energies above $1 \mathrm{MeV}$ and SP2 spherical proton recoil proportional counters for neutron energies between a few $\mathrm{keV}$ and $2 \mathrm{MeV}$ [3].

Since the total and differential cross section of the neutron reaction on the hydrogen is very well known (about 1\%) proton recoil proportional counter are very well suited for neutron fluence determination [4]. To determine precisely the response function including wall and electric field effects, the best way is to use simulation.

In order to simulate the response energy distribution of the proportional counter, the MCNPX code [5] can be used. The advantage of this code is the possibility to describe in a very detailed way the counter geometry. However, the electric field effect inside the detector is not included as in the SPHERE code [6].

The goal of this study is to develop a method to include the electric field effect by weighting the deposited energy by protons in the MCNPX simulations. The modeling of the electric field effect inside the SP2 counter by a dedicated a program will first be presented as well as the method to take into account the electric field effect within the MCNPX calculation. Finally, the comparison between the simulated and the experimental responses will be discussed.

\section{Modeling of the response energy distribution of a spherical counter with MCNPX}

\subsection{Geometrical parameters of the counter}

The studied detector is a SP2 spherical proton recoil proportional counter of $20.04 \mathrm{~mm}$ radius filled with 3 atmosphere of pure hydrogen. It works for incident neutron energies included between $100 \mathrm{keV}$ and $1 \mathrm{MeV}$. The voltage applied to the $25 \mu \mathrm{m}$ diameter wire anode is equal to $1860 \mathrm{~V}$. Recoil protons are generated by elastic scattering of the neutrons on hydrogen and lose their energy through the creation of ion-electron pairs all along their range in the gas mixture. The electrons are accelerated in the anode direction by the electric field. A multiplication of the charges (gas amplification) occurs by avalanche close to the anode. The deposited proton energy is proportional to the collected charges. The figure 1 (left) shows in 3 dimensions the counter geometry created in MCNPX.

\subsection{Determination of the electric field inside the counter}

To determine the electric field effect inside the counter, the method described by Weise et al. in [7] has been used. In a first step, the electric potential is determined from the Laplace's equation discretizised into a set of linear equations and solved by using a Gauss-Seidel iterative method [8]. 
Once the potential is known, the electric field close to the anode wire can be deduced from the Maxwell's equation in a cylindrical system. The whole electric field lines are reconstructed by just connecting the electric field, calculated at the anode wire, i.e. a vector, to a scalar value for each detector point. To realize this connection, the Gauss's law is used. From this method, a home made program has been written which provides a matrix giving the electric field values for each point describing the counter geometry.
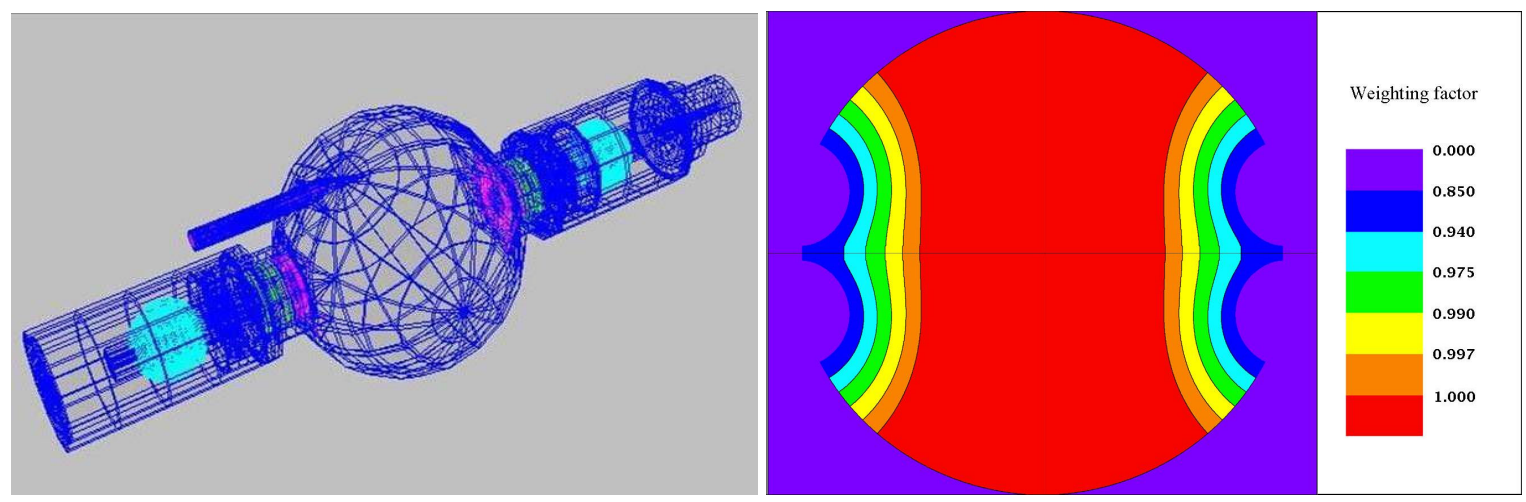

Fig. 1: Left: 3D vision of the counter. Right: gas amplification weighting factor inside a SP2 counter filled with 3 atmosphere of pure hydrogen and an anode high voltage of $1860 \mathrm{~V}$ (right).

\subsection{Electric field integration in the MCNPX simulation}

The electric field effect has to be implemented in the MCNPX simulation of the proportional spherical counter response energy distribution. The electric field influences the gas amplification, and then, the deposited energy by recoil protons. If the electric field is distorted in some detector area, the gas amplification can be there lower than expected, leading to more events with low deposited energy and less events at the high deposited energies which generates an increase of the slope of this response. The used method is to take into account the gas amplification and not the electric field directly. Coefficients corresponding to the gas amplification variation into the counter can be deduced from a calculated matrix of the electric field and the formula described in references [9][10].

In this matrix, the electric field lines define area with different gas amplification value. The gas amplification in each zone is divided by the maximum obtained at the center of the counter leading to a weighting factor between 0 and 1 for each zone. The resulting normalized matrix is represented in the figure 1 (right).

The implementation method consists in creating cells described by overlapping torus in MCNPX geometry associated to the gas amplification zones and to apply the corresponding weighting factor to the energy deposited in these zones. To create the cells, The Moritz software [11] has been used, adjusting the torus parameters to a background picture of the normalized amplification gas matrix. 
The deposited proton energy is multiplied in each so defined cell of the active volume by the gas amplification weighting factor. The F8 tally method with the FT PHL option is used to achieve this calculation. This option ties the F8 tally in one or more energy deposition F6 tallies. If a track comes through the set of the active volume cells, the F6 tally will be multiplied in each area by the corresponding weighting factor. This multiplication is executed by the FM option. The corrected deposited energies in each area are then added and the total resulting energy is putted in the corresponding E8 energy bin. MCNPX simulations with and without electric field effect are compared in the figure 2 at $144 \mathrm{keV}$ and $565 \mathrm{keV}$ showing the importance of taking this feature into account.
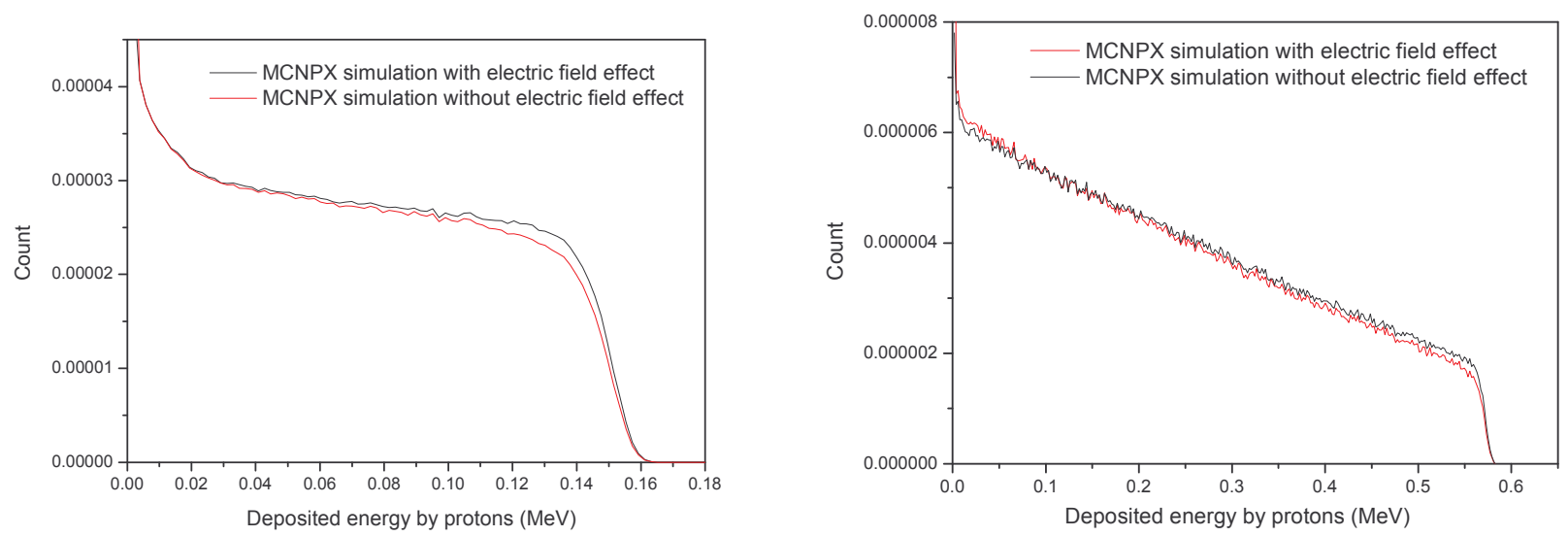

Fig. 2: MCNPX simulated responses to the experimental data at $144 \mathrm{keV}$ and $565 \mathrm{keV}$ with and without electric field effect.

\section{Comparison between the response energy distribution simulation including the electric field effect and experimental data.}

The response energy distribution calculated by MCNPX is then compared to the SPHERE code calculation and experimental data obtained at the PTB reference mono-energetic neutron fields with the 0.3 MPa hydrogen filled SP2 [3]. The neutron energy and fluence were calculated with the PTB reference instruments. The neutron fluence energy distribution has been determined by the TARGET code [12] using the following parameters: neutrons were produced in a $70 \mu \mathrm{g} / \mathrm{cm}^{2}$ thick LiF target using ${ }^{7} \mathrm{Li}(\mathrm{p}, \mathrm{n})^{7} \mathrm{Be}$ reaction and the detector was placed at $52.05 \mathrm{~cm}$ from the target and at $0^{\circ}$ with respect to the beam axis. The high voltage of the counter was set to $1860 \mathrm{~V}$. The background contribution coming from scattered neutrons was determined using an appropriate shadow cone between neutron source and detector. This contribution was substracted to obtain the neutron spectral fluence emitted by the target. Two mono-energetic neutron fields have been chosen for this study: $144 \mathrm{keV}$ and $565 \mathrm{keV}$. In the first case, there are only short ranges of proton recoil into the detector, whereas at $565 \mathrm{keV}$ energy, long ranges are also available. 

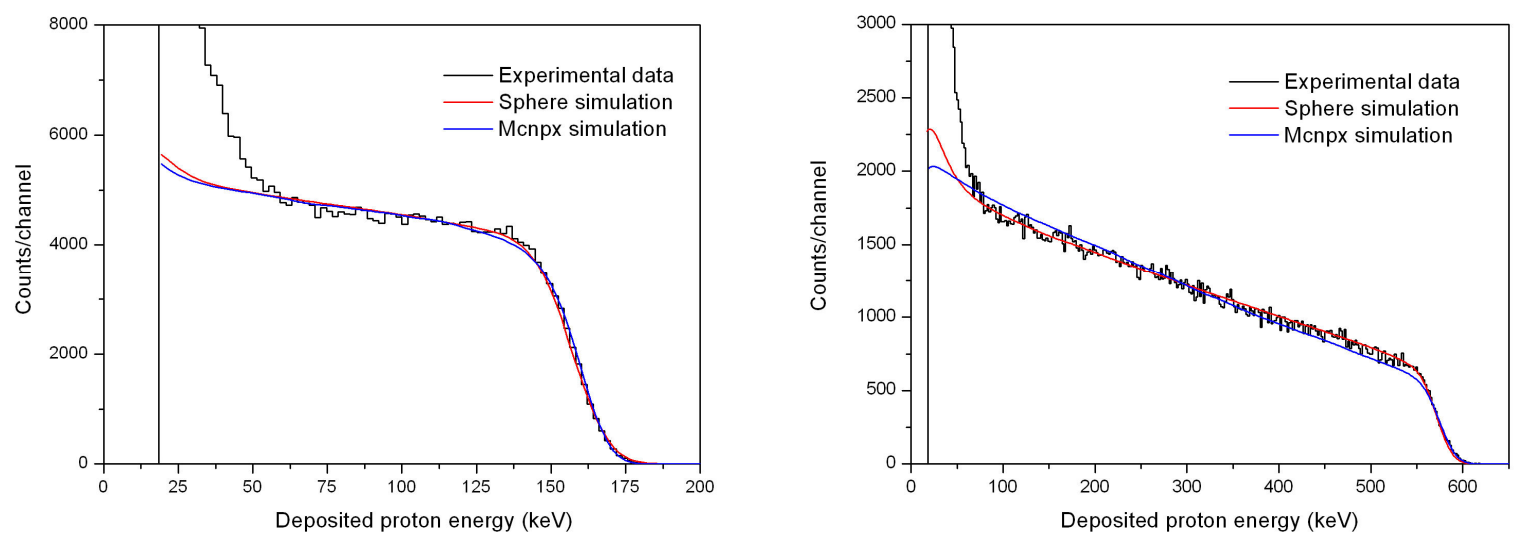

Fig. 3: Adjustment of the SP2 simulated responses to the experimental data at $144 \mathrm{keV}$ and $565 \mathrm{keV}$.

The method used to compare the detector responses obtained by simulations (SPHERE and MCNPX) with the experimental data, presents several steps. Simulations are adjusted to the experimental using both codes Spkflt and Spkfit [13]. The adjusted parameters are energy resolution and energy calibration. The results of the comparisons between the data and the simulations are shown in the figure 3.

At $144 \mathrm{keV}$, simulations with MCNPX and SPHERE are similar and in relatively good agreement with the experimental data. At $565 \mathrm{keV}$, the SPHERE calculation reproduces perfectly the measurements, whereas there is a noticeable discrepancy between MCNPX response simulation slope and the SPHERE simulation and experimental slopes. This disagreement has been investigated and can be explained by the use of different proton range-energy tables in each code.

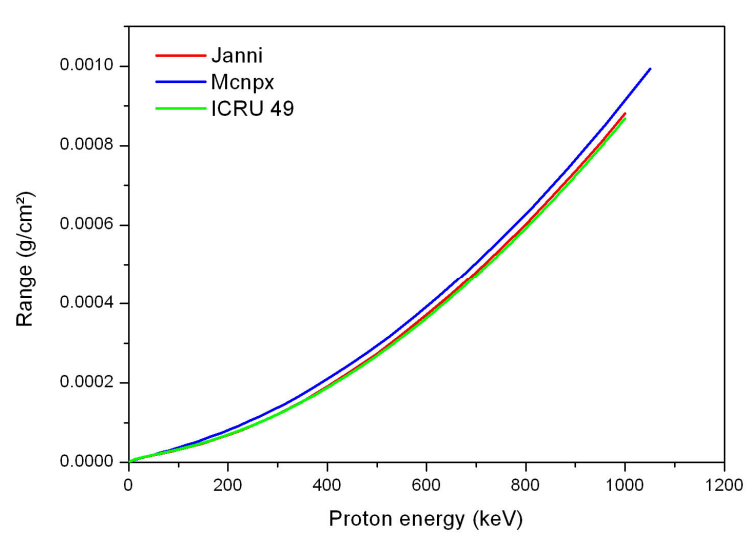

Fig. 4: Ranges of protons in pure $\mathrm{H}_{2}$ gas at several energies. The data are coming from three different tables: MCNPX, Janni's tables used by SPHERE and ICRU 49.

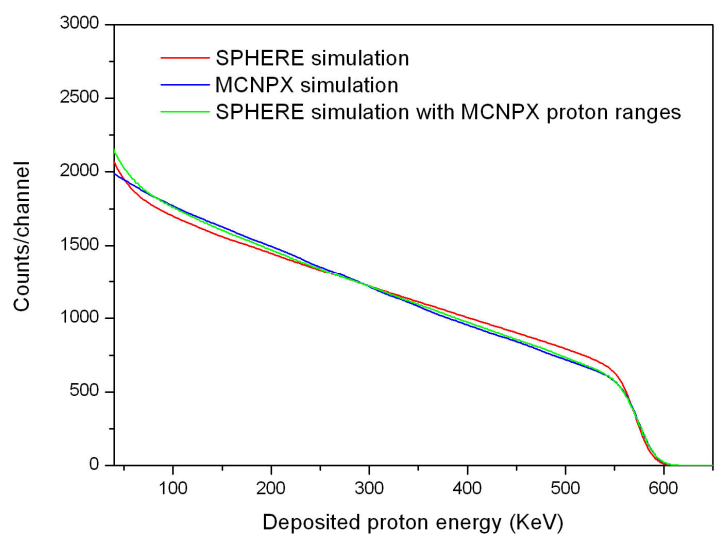

Fig. 5: Comparison berween the MCNPX simulation, the SPHERE simulation and the SPHERE simulation at $565 \mathrm{keV}$ using the MCNPX proton ranges. 
In MCNPX, the proton range is calculated from SPAR code methodology [14] whereas in SPHERE code, the Janni's proton range tables [15] are used. The figure 4 compares these proton ranges in pure $\mathrm{H} 2$ gas with the reference data issued from ICRU 49 [16]. It appears that Janni's data and the ICRU data are very close. However, the MCNPX range data are several percents higher than the two others.

Performing SPHERE simulation with MCNPX proton range-energy tables leads to very comparable results to MCNPX simulation as shown in the figure 5. This proves that the deviation observed with the experimental response energy distribution at $565 \mathrm{keV}$ is due to the proton range calculation in MCNPX. As a conclusion, the use of MCNPX to calculate the response energy distributions of proportional counters could not be performed properly until the ICRU 49 proton range tables are not included in the code.

\section{Conclusion and perspectives}

The spherical proportional counters SP2 are used to determine the references of the AMANDE facility neutron fields between few $\mathrm{keV}$ and $2 \mathrm{MeV}$. The use of MCNPX to calculate the response matrices of these counters has been investigated. MCNPX presents the advantage to be able to describe in more detail the geometry of the counters and their environment. In this study, a method to implement the electric field effect in the simulations has been developed. However, comparisons with the SPHERE code and experimental data have shown differences. Whereas SPHERE simulation fit well the measurements, MCNPX simulation deviates from the experimental data, especially in the case of long proton ranges into the detector. The problem has been explained from differences in the proton range calculation in MCNPX compare to the reference data from ICRU 49. Until this correction is performed, SPHERE is still the most suitable simulation code for spherical proportional counter response matrix calculation. Calculation with MCNPX and experimental studies will however continue, especially concerning $(n, p)$ reactions of high energy neutrons $\left(E_{n}>4 \mathrm{MeV}\right)$ into the detector walls [17].

\section{Acknowledgments}

The authors are grateful to Dr. Veronique Lacoste for her valuable help concerning the proton recoil detector response simulation with the MCNPX code.

\section{References}

[1] V. Gressier, G. Pelcot, J.L. Pochat, T. Bolognese-Milstajn, New IRSN facilities for neutron production, Nucl. Instr. And Meth. A505 (2003) 370-373.

[2] S. Schmidt, B. Asselineau, R. Böttger, H. Klein, L. Lebreton, S. Neumann, R. Nolte, G. Pichenot, Characterization of liquid scintillation detector, Nucl. Instr. and Meth. A476 (2002) 186-189.

[3] G. Pichenot et al., Characterisation of spherical recoil proton proportional counters used for neutron spectrometry, Nucl. Instr. And Meth A476 (2002) 165-169.

[4] M. Weyrauch and K. Knauf, Absolute neutron fluence determination with a spherical proton recoil proportional counter, Rad. Prot. Dosim. Vol 44 (1992) 97-99. 
[5] L.S. Waters, Ed. MCNPX - User's manual, version 2.5.0, Los Alamos National Laboratory report. LA-CP-05-0369 (2005).

[6] K. Knauf, M. Weyrauch, F. Simon and N. Kaldune, SPHERE - A program Package for calculating the neutron response of spherical proton recoil proportional counters, PTB-6.42-98-1 report (1998).

[7] K. Weise, M. Weyrauch and K. Knauf, Neutron response of a spherical proton recoil proportional counter, Nucl. Instr. And Meth. A309 (1991) 287-293.

[8] W.H. Press, S.A. Teukolsky, W.T. Vetterling and B.P. Flannery, Numerical Recipes in FORTRAN, the art of scientific computing, $2^{\text {nd }}$ edition, Cambridge University Press (1989).

[9] W. Diethorn, A methane proportional counter system for natural radiocarbon measurements, USAEC report NY06628 (1956) .

[10] G.F. Knoll, (third edition). Radiation detection and measurement, John Wiley \& sons Inc., New York (2000).

[11] K.A. Van Riper, Moritz - User's guide, White Rock Science.

[12] D. Schlegel, TARGET - User's manual, PTB-6.41-98-1 report (1998).

[13] G. Dietze, A dialog program for multichannel spectra analysis, PTB-ND-13 report (1978).

[14] T.W. Armstrong and K.D. Chandler, SPAR, a fortran program for computing stopping powers and ranges for muons, charged pions, protons and heavy ions, Oak Ridge National Laboratory report ORNL-4869 (1973).

[15] J. JANNI, A proton range-energy tables, $1 \mathrm{keV}-10 \mathrm{GeV}$ energy loss, range, path length, time-offlight, straggling, multiple scattering, and nuclear interaction probability, Atomic Data and Nuclear Tables vol 27 (1982) 341-529.

[16] International Commission on Radiation Units and Measurements, ICRU report 49, stopping powers and ranges for protons and alpha particles, ICRU report 49 (1993).

[17] J.S. Petler and M.C. Scott, The effect of charged particles produced in detector walls on the use of proton recoil proportional counters for neutron spectrometry, Nucl. Instr. And Meth. 228 (1985) 425-431. 